\title{
Superconducting 2D System with Lifted Spin Degeneracy: Mixed Singlet-Triplet State
}

\section{Citation}

Gor'kov, Lev P., and Emmanuel I. Rashba. 2001. "Superconducting 2D System with Lifted Spin Degeneracy: Mixed Singlet-Triplet State." Physical Review Letters 87 (3) (July 2). doi:10.1103/ physrevlett.87.037004.

\section{Published Version}

doi:10.1103/PhysRevLett.87.037004

\section{Permanent link}

http://nrs.harvard.edu/urn-3:HUL.InstRepos:25875692

\section{Terms of Use}

This article was downloaded from Harvard University's DASH repository, and is made available under the terms and conditions applicable to Other Posted Material, as set forth at http:// nrs.harvard.edu/urn-3:HUL.InstRepos:dash.current.terms-of-use\#LAA

\section{Share Your Story}

The Harvard community has made this article openly available.

Please share how this access benefits you. Submit a story.

\section{Accessibility}




\title{
Superconducting 2D System with Lifted Spin Degeneracy: Mixed Singlet-Triplet State
}

\author{
Lev P. Gor'kov* \\ National High Magnetic Field Laboratory, Florida State University, Tallahassee, Florida 32310 \\ Emmanuel I. Rashba ${ }^{\dagger}$ \\ Department of Physics, The State University of New York at Buffalo, Buffalo, New York 14260
}

(Received 20 March 2001; published 2 July 2001)

\begin{abstract}
Motivated by recent experimental findings, we have developed a theory of the superconducting state for $2 \mathrm{D}$ metals without inversion symmetry modeling the geometry of a surface superconducting layer in a field-effect transistor or near the boundary doped by adsorbed ions. In such systems the twofold spin degeneracy is lifted by spin-orbit interaction, and singlet and triplet pairings are mixed in the wave function of the Cooper pairs. As a result, spin magnetic susceptibility becomes anisotropic and Knight shift retains finite and rather high value at $T=0$.
\end{abstract}

DOI: $10.1103 /$ PhysRevLett.87.037004

In this presentation we consider the energy spectrum and some other properties of the superconducting state for a two-dimensional (2D) system without inversion symmetry when the twofold degeneracy of the electron energy spectrum is lifted by spin-orbit ( $\mathrm{SO}$ ) interaction. We have been motivated by several recent experimental discoveries. Superconductivity (SC) with critical temperature $T_{c}=91 \mathrm{~K}$ existing in small islands at the surface of insulating $\mathrm{WO}_{3}$ (when the surface was doped by $\mathrm{Na}^{+}$ions) was reported in Ref. [1]. We speculate that strong near-surface electric fields should be present in the surface layer of these crystals quite similar to the 2D superconducting fullerene and polyacene crystals in the field-effect-transistor geometry [2]. In centrosymmetric crystals the 3D inversion ensures twofold degeneracy of the energy spectrum throughout the Brillouin zone both in the normal [3] and in SC [4] states. In SC state this symmetry allows classification of Cooper pairs by their parity (although spin and orbital degrees of freedom are already mixed by SO interaction [4]). If 3D inversion is broken by the space inhomogeneity of the order parameter, mixing of the singlet and triplet states (or, more rigorously, states of different parity) becomes possible $[5,6]$. The twofold degeneracy of the spectrum is always broken in the surface layer, even homogeneous, because of the SO coupling and violation of 3D inversion. This effect is known and well documented experimentally $[7,8]$ in physics of semiconductor microstructures, and has been recently discovered in surface states of metallic Au [9] and also of Mo and W covered by Li [10].

It is known that split-off $2 \mathrm{D}$ electron bands may form at the surface (Tamm levels). Lowering of the symmetry in the surface layer results in a new SO invariant of the group $\mathrm{C}_{\infty \mathrm{V}}$ (and its discrete subgroups) in the free electron Hamiltonian

$$
H_{\mathrm{so}}=\alpha(\boldsymbol{\sigma} \times \mathbf{p}) \cdot \mathbf{n}
$$

dubbed in the literature as Rashba term [11,12]. Here $\mathbf{p}$ is a $2 \mathrm{D}$ quasimomentum, $\boldsymbol{\sigma}$ are Pauli matrices, and $\mathbf{n}$ is a unit vector normal to the surface $(\hbar=1)$. Despite the fact
PACS numbers: 74.20.-z, 71.18.+y, 73.20.At, 76.60.Cq

that $\mathbf{p}$ is two-dimensional, the 3D group controls the symmetry of the system since $\mathbf{n}$ determines the preferred direction of the normal to the plane and spins are always 3D. Total single electron Hamiltonian, $H=H_{0}+H_{\text {so }}$, diagonalizes in eigenfunctions $\eta_{\lambda}(\mathbf{p})$ and eigenvalues $\varepsilon_{\lambda}(p)$,

$$
\begin{gathered}
\eta_{\lambda}(\mathbf{p})=\frac{1}{\sqrt{2}}\left(\begin{array}{c}
1 \\
i \lambda \exp \left(i \varphi_{\mathbf{p}}\right)
\end{array}\right), \\
\varepsilon_{\lambda}(p)=\varepsilon_{0}(p)+\varepsilon_{\lambda}^{\mathrm{so}}(p), \quad \varepsilon_{\lambda}^{\mathrm{so}}(p)=-\alpha \lambda p,
\end{gathered}
$$

$\lambda= \pm 1$, and the Fermi surface splits into two sheets. SO interaction $H_{\text {so }}$ puts electron spins into the plane, $(\perp \mathbf{n})$, where they are aligned perpendicular to the quasimomentum $\mathbf{p}$. The magnitude of the coefficient $\alpha$ depends on the electric field, presence of adatoms at the boundary, atomic weight, and atomic shells involved [13]. Angle resolved photoemission spectroscopy data on the $s-p$ surface band of $\mathrm{Au}$ are in excellent agreement with Eq. (2). They result in a nearly isotropic spectrum with $\alpha \approx 5 \times 10^{-9} \mathrm{eV}$. $\mathrm{cm}$ (that is, somewhat larger than in typical semiconductor systems) and in the SO splitting at the Fermi level $2|\alpha| p_{F}=0.11 \mathrm{eV}$ [9]. Anisotropic $d$-derived bands of Mo and W show even larger SO splittings up to 0.13 and $0.5 \mathrm{eV}$, respectively, depending on the surface concentration of adsorbed $\mathrm{Li}^{+}$ions.

In what follows we investigate specific properties of BCS-type 2D SCs caused by lifting the electron spin degeneracy in the presence of the SO interaction. This problem has been addressed previously by Bulaevskii et al. [14] who investigated magnetic properties of layered and pyroelectric metals and by Edelstein [15] who, inter alia, predicted magnetoelectric effect in low-symmetry SCs and discussed the possible role of SO effects in layered high- $T_{c}$ cuprates. We find the explicit form of the wave function of Cooper pairs, including its singlet and triplet components, and demonstrate the dramatic effect of the symmetry breaking on spin magnetic susceptibility and Knight shift. 
For the sake of simplicity we consider a two-particle interaction $U\left(\left|\mathbf{r}-\mathbf{r}^{\prime}\right|\right)$ (here and below all vectors lie in the plane). Retardation effects such as due to the phonon mediated attraction are known to be of no importance for a weak coupling scheme. To put it briefly, we do not address at all the issue of the mechanism responsible for pairing, and restrict ourselves by features pertaining to the reduced dimensionality (for a proper symmetry at the surface) and spin-split isotropic electron spectrum. The SO splitting at the Fermi level, $2 \alpha p_{F}$ (here and below $\alpha>0$ ), can be comparable to, or exceed, the SC characteristic scale, the critical temperature $T_{c}$. However, it is assumed to be small compared to the Fermi energy, $2 \alpha p_{F} \ll \varepsilon_{F}$, and $p_{+}-$ $p_{-} \ll p_{F}$, where $p_{\lambda}=p_{ \pm}$are Fermi momenta for the two SO split sheets of the Fermi surface. Therefore, near the Fermi energy the electron dispersion is taken linear,

$$
\xi_{\lambda}(p)=v_{F}\left(p-p_{\lambda}\right), \quad p_{\lambda}=\left(1+\alpha \lambda / v_{F}\right) p_{F} .
$$

The total electron Hamiltonian $H=H_{0}+H_{\text {so }}+H_{\text {int }}$ includes the interaction Hamiltonian

$$
\begin{aligned}
H_{\text {int }}= & \frac{1}{2} \sum_{\lambda \mu \nu \rho} \sum_{\mathbf{p} \mathbf{p}^{\prime} \mathbf{q}} U_{\lambda \mu \nu \rho}\left(\mathbf{p}, \mathbf{p}^{\prime}, \mathbf{q}\right) \\
& \times a_{\lambda}^{+}(\mathbf{p}) a_{\mu}^{+}(-\mathbf{p}-\mathbf{q}) a_{\nu}\left(-\mathbf{p}^{\prime}-\mathbf{q}\right) a_{\rho}\left(\mathbf{p}^{\prime}\right) .
\end{aligned}
$$

Here $a_{\lambda}(\mathbf{p})$ are Fermi electronic operators, and $U_{\lambda \mu \nu \rho}\left(\mathbf{p}, \mathbf{p}^{\prime}, \mathbf{q}\right)$ is the interaction potential written in the representation of the spinors $\eta_{\lambda}(\mathbf{p})$

$$
\begin{aligned}
U_{\lambda \mu \nu \rho}\left(\mathbf{p}, \mathbf{p}^{\prime}, \mathbf{q}\right)= & U\left(\left|\mathbf{p}-\mathbf{p}^{\prime}\right|\right)\left[\eta_{\lambda}(\mathbf{p}), \eta_{\rho}\left(\mathbf{p}^{\prime}\right)\right] \\
& \times\left[\eta_{\mu}(-\mathbf{p}-\mathbf{q}), \eta_{\nu}\left(-\mathbf{p}^{\prime}-\mathbf{q}\right)\right],
\end{aligned}
$$

$U(|\mathbf{p}|)$ is the Fourier image of the interaction potential, and scalar products of spinors are equal to

$$
\left[\eta_{\lambda}(\mathbf{p}), \eta_{\lambda^{\prime}}\left(\mathbf{p}^{\prime}\right)\right]=\frac{1}{2}\left\{1+\lambda \lambda^{\prime} \exp \left[-i\left(\varphi_{\mathbf{p}}-\varphi_{\mathbf{p}^{\prime}}\right)\right]\right\} .
$$

In what follows we employ the method of thermodynamic (Matsubara) Green functions [16]. Onset of the SC state manifests itself as the appearance, side by side with the regular Green functions [17]

$$
g_{\lambda}\left(\mathbf{p}, \tau-\tau^{\prime}\right)=-\left\langle T\left\{\tilde{a}_{\lambda}(\mathbf{p}, \tau) \tilde{a}_{\lambda}^{+}\left(\mathbf{p}, \tau^{\prime}\right)\right\}\right\rangle,
$$

also of the nonzero anomalous (Gor'kov) averages [18]

$$
\begin{aligned}
f_{\lambda}\left(\mathbf{p}, \tau-\tau^{\prime}\right) & =\lambda\left\langle T\left\{\tilde{a}_{\lambda}(\mathbf{p}, \tau) \tilde{a}_{\lambda}\left(-\mathbf{p}, \tau^{\prime}\right)\right\}\right\rangle, \\
f_{\lambda}^{+}\left(\mathbf{p}, \tau-\tau^{\prime}\right) & =\lambda\left\langle T\left\{\tilde{a}_{\lambda}^{+}(\mathbf{p}, \tau) \tilde{a}_{\lambda}^{+}\left(-\mathbf{p}, \tau^{\prime}\right)\right\}\right\rangle,
\end{aligned}
$$

where operators $\tilde{a}_{\lambda}(\mathbf{p}, \tau)$ are in the Heisenberg representation. Functions $f_{\lambda}(\mathbf{p}) \equiv f_{\lambda}(\mathbf{p}, 0+)$ that obey the relation

$$
f_{\lambda}^{*}(\mathbf{p})=f_{\lambda}^{+}(-\mathbf{p})
$$

are components of the wave functions of Cooper pairs at each spectrum branch. Fermionic commutation relations result in

$$
f_{\lambda}(\mathbf{p})=-f_{\lambda}(-\mathbf{p}) .
$$

From equations of motion for the operators $\tilde{a}_{\lambda}(\mathbf{p}, \tau)$, $\partial_{\tau} \tilde{a}_{\lambda}(\mathbf{p}, \tau)=\left[H, \tilde{a}_{\lambda}(\mathbf{p}, \tau)\right]$, equations of motion for $g_{\lambda}\left(\mathbf{p}, \tau-\tau^{\prime}\right)$ and $f_{\lambda}\left(\mathbf{p}, \tau-\tau^{\prime}\right)$ follow in the usual way. They include four-fermion $T$ products, the standard decoupling $[16,18]$ of which results in

$$
\begin{gathered}
{\left[\partial_{\tau}+\varepsilon_{\lambda}(p)\right] g_{\lambda}\left(\mathbf{p}, \tau-\tau^{\prime}\right)-\lambda \sum_{\mu \mathbf{p}^{\prime}} \mu U_{\lambda \lambda \mu \mu}\left(\mathbf{p}, \mathbf{p}^{\prime}, 0\right) f_{\mu}\left(-\mathbf{p}^{\prime}\right) f_{\lambda}^{+}\left(-\mathbf{p}, \tau-\tau^{\prime}\right)=-\delta\left(\tau-\tau^{\prime}\right),} \\
{\left[\partial_{\tau}-\varepsilon_{\lambda}(p)\right] f_{\lambda}^{+}\left(-\mathbf{p}, \tau-\tau^{\prime}\right)+\lambda \sum_{\mu \mathbf{p}^{\prime}} \mu U_{\mu \mu \lambda \lambda}\left(\mathbf{p}^{\prime}, \mathbf{p}, 0\right) f_{\mu}^{+}\left(\mathbf{p}^{\prime}\right) g_{\lambda}\left(\mathbf{p}, \tau-\tau^{\prime}\right)=0 .}
\end{gathered}
$$

For the Cooper pairs with the zero total momentum forming the condensate, $\mathbf{q}=0$ in $U_{\lambda \lambda \mu \mu}\left(\mathbf{p}, \mathbf{p}^{\prime}, \mathbf{q}\right)$. Next simplification in the potential comes from the fact that it enters in Eqs. (12) and (13) only in the combination with the functions $f_{\mu}(\mathbf{p})$; hence, because of Eq. (11), $U_{\lambda \lambda \mu \mu}\left(\mathbf{p}, \mathbf{p}^{\prime}, 0\right)$ should be antisymmetrized. Simple algebra shows

$$
\begin{aligned}
U_{\lambda \mu}\left(\mathbf{p}, \mathbf{p}^{\prime}\right) & \equiv \frac{1}{2}\left[U_{\lambda \lambda \mu \mu}\left(\mathbf{p}, \mathbf{p}^{\prime}, 0\right)-U_{\lambda \lambda \mu \mu}\left(\mathbf{p},-\mathbf{p}^{\prime}, 0\right)\right] \\
& \approx \frac{1}{2} \lambda \mu U(0) \exp \left[i\left(\varphi_{\mathbf{p}^{\prime}}-\varphi_{\mathbf{p}}\right)\right] .
\end{aligned}
$$

Having in mind $s$ pairing we neglected the momentum dependence of the potential in the right hand side of Eq. (14). Making Fourier transformation of Eqs. (12) and (13) to the Matsubara frequencies $i \omega_{n}$, we find the final form of Gor'kov equations

$$
\left[i \omega_{n}-\varepsilon_{\lambda}(\mathbf{p})\right] g_{\lambda}\left(\mathbf{p}, \omega_{n}\right)+\Delta(\mathbf{p}) f_{\lambda}^{+}\left(-\mathbf{p}, \omega_{n}\right)=1,
$$

$$
\Delta^{+}(\mathbf{p}) g_{\lambda}\left(\mathbf{p}, \omega_{n}\right)+\left[i \omega_{n}+\varepsilon_{\lambda}(\mathbf{p})\right] f_{\lambda}^{+}\left(-\mathbf{p}, \omega_{n}\right)=0,
$$

where the "gap" $\Delta(\mathbf{p})$ is

$$
\begin{aligned}
\Delta(\mathbf{p}) & =\exp \left(-i \varphi_{\mathbf{p}}\right) \Delta_{0}, \\
\Delta_{0} & =\frac{1}{2} U(0) \sum_{\lambda \mathbf{p}} \exp \left(i \varphi_{\mathbf{p}}\right) f_{\lambda}(\mathbf{p}), \\
\Delta^{+}(\mathbf{p}) & =\Delta^{*}(\mathbf{p}) .
\end{aligned}
$$

Before we turn to the discussion of the nature of the order parameter in the new SC state, let us mention that the "gap function" $\Delta(\mathbf{p})$ depends on $\mathbf{p}$ through its phase. This dependence is inherent in the nonperturbative character of is lifted. It cannot be eliminated but can be changed by a different choice of phase factors in Eq. (2). Green functions diagonal in branch indices are [17] the spinor basis functions of Eq. (2) after spin degeneracy 


$$
\begin{aligned}
g_{\lambda}\left(\mathbf{p}, \omega_{n}\right) & =-\left[i \omega_{n}+\xi_{\lambda}(\mathbf{p})\right] / D_{n \lambda}(\mathbf{p}), \\
f_{\lambda}^{+}\left(-\mathbf{p}, \omega_{n}\right) & =\Delta^{+}(\mathbf{p}) / D_{n \lambda}(\mathbf{p}), \\
D_{n \lambda}(\mathbf{p}) & =\omega_{n}^{2}+\xi_{\lambda}^{2}(\mathbf{p})+|\Delta|^{2} .
\end{aligned}
$$

The self-consistency condition for $|\Delta(\mathbf{p})| \equiv \Delta(T)$ is

$$
-\frac{1}{2} T U(0) \sum_{n \lambda \mathbf{p}} D_{n \lambda}^{-1}(\mathbf{p})=1 .
$$

In the approximation $2 \alpha p_{F} \ll \varepsilon_{F}$, the left hand side of Eq. (19) does not depend on $\alpha$ and coincides with the BCS equation for $\alpha=0$. The energy spectrum found from the poles of $g_{\lambda}\left(\mathbf{p}, \omega_{n}\right)$ and $f_{\lambda}\left(\mathbf{p}, \omega_{n}\right)$ is

$$
E_{\lambda}(p)= \pm\left[v_{F}^{2}\left(p-p_{\lambda}\right)^{2}+\Delta^{2}(T)\right]^{1 / 2} ;
$$

i.e., it consists of two gapped branches. One sees that weak SO coupling makes no changes in thermodynamical properties of the new BCS-like state. In particular, the relation $\Delta(0)=(\pi / \gamma) T_{c} \approx 1.76 T_{c}$ holds in this case.

The Green functions were found above in the representation of energy branches indices $\lambda$. To get insight on the symmetry of the new SC state we rewrite them as matrices $\hat{G}\left(\mathbf{p}, \omega_{n}\right)$ and $\hat{F}\left(\mathbf{p}, \omega_{n}\right)$ in the basis of the components $\eta_{\lambda}^{\alpha}(\mathbf{p})$ of the spinors $\eta_{\lambda}(\mathbf{p})$,

$$
\begin{aligned}
G_{\alpha \beta}\left(\mathbf{p}, \omega_{n}\right) & =\sum_{\lambda} \eta_{\lambda}^{\alpha}(\mathbf{p}) g_{\lambda}\left(\mathbf{p}, \omega_{n}\right) \eta_{\lambda}^{\beta}(\mathbf{p})^{*} \\
F_{\alpha \beta}\left(\mathbf{p}, \omega_{n}\right) & =\sum_{\lambda} \eta_{\lambda}^{\alpha}(\mathbf{p}) f_{\lambda}\left(\mathbf{p}, \omega_{n}\right) \eta_{\lambda}^{\beta}(\mathbf{p}),
\end{aligned}
$$

and split $g_{\lambda}\left(\mathbf{p}, \omega_{n}\right)$ and $f_{\lambda}\left(\mathbf{p}, \omega_{n}\right)$ into their symmetric and antisymmetric in $\lambda$ parts,

$$
g_{\lambda}=g_{\mathrm{s}}+\frac{1}{2} \lambda g_{\mathrm{as}}, \quad f_{\lambda}=f_{\mathrm{s}}+\frac{1}{2} \lambda f_{\text {as }} .
$$

Equation (22) implies that $\hat{G}=\hat{G}_{\mathrm{s}}+\hat{G}_{\text {as }}$ and $\hat{F}=\hat{F}_{\mathrm{s}}+$ $\hat{F}_{\text {as }}$. Antisymmetric parts of $g_{\lambda}$ and $f_{\lambda}$ (and also of $\hat{G}$ and $\hat{F}$ ) originate because of the splitting of the energy spectrum by the SO interaction. It is convenient to write $\hat{G}_{\mathrm{s}}$ and $\hat{G}_{\text {as }}$ (and also $\hat{F}_{\mathrm{S}}$ and $\hat{F}_{\text {as }}$ ) in terms of the Pauli matrices $\boldsymbol{\sigma}$ and the unit matrix $\sigma_{0}$. After some algebra we get

$$
\begin{gathered}
\hat{G}_{\mathrm{s}}\left(\mathbf{p}, \omega_{n}\right)=g_{\mathrm{s}}\left(\mathbf{p}, \omega_{n}\right) \sigma_{0}, \\
\hat{G}_{\text {as }}\left(\mathbf{p}, \omega_{n}\right)=\frac{1}{2} g_{\text {as }}\left(\mathbf{p}, \omega_{n}\right)\left(\mathbf{p}^{0} \times \boldsymbol{\sigma}\right) \cdot \mathbf{n},
\end{gathered}
$$

$$
\begin{gathered}
\hat{F}_{\mathrm{s}}\left(\mathbf{p}, \omega_{n}\right)=\left[f_{\mathrm{s}}\left(\mathbf{p}, \omega_{n}\right) e^{i \varphi_{\mathbf{p}}}\right] \sigma_{y}, \\
\hat{F}_{\mathrm{as}}\left(\mathbf{p}, \omega_{n}\right)=-\left[f_{\mathrm{as}}\left(\mathbf{p}, \omega_{n}\right) e^{i \varphi_{\mathbf{p}}}\right] \sigma_{y}\left(\boldsymbol{\sigma} \cdot \mathbf{p}^{0}\right),
\end{gathered}
$$

where $\mathbf{p}^{0}=\mathbf{p} / p$. Phase factors $e^{i \varphi(\mathbf{p})}$ drop out from both $\hat{F}_{\mathrm{S}}$ and $\hat{F}_{\text {as }}$ according to Eqs. (17) and (18). $\hat{G}_{\text {as }}$ and $\hat{F}_{\text {as }}$ components of the Green functions are of the order of unity but only inside a narrow ring of the momentum space, $\Delta p \approx p_{+}-p_{-}$, between two Fermi surfaces.

Both contributions to $\hat{G}$ are invariants of the group $\mathrm{C}_{\infty \mathrm{v}}$; however, they are of opposite parity if the transformation $\mathbf{p} \rightarrow-\mathbf{p}$, rotation by $\pi$ around the $z$ axis, is performed only in coordinate space with spin remaining unchanged. Both $\hat{G}_{\text {as }}$ and $\hat{F}_{\text {as }}$ depend on the azimuth of $\mathbf{p}^{0}$. The factor $\left(\boldsymbol{\sigma} \cdot \mathbf{p}^{0}\right)$ is a pseudoscalar to the $3 \mathrm{D}$ inversion. We attribute to $\hat{G}_{\text {as }}$ a number of transport effects in normal conductors with lifted spin degeneracy which have been observed or predicted.

The existence of nonzero $\hat{F}$ function, Eqs. (25) and (26), means the broken gauge symmetry in the SC state, $U(1)$. Equation (11), when expressed in terms of the functions $F_{\alpha \beta}\left(\mathbf{p}, \omega_{n}\right)$, reflects permutational symmetry of the wave function of the two electrons of the pair, $(\mathbf{p}, \alpha)$ and $(-\mathbf{p}, \beta)$. If there is the $3 \mathrm{D}$ inversion center, one can make the total wave function odd either by choosing an antisymmetric spin wave function while the space part is symmetric (singlet pairing, $S=0$ ), or, vice versa, by choosing a symmetric spin wave function while the space part is antisymmetric (triplet pairing, $S=1$ ); see Ref. [4]. Therefore, $F_{\alpha \beta} \propto\left(\sigma_{y}\right)_{\alpha \beta}$ for $S=0$ and $F_{\alpha \beta} \propto$ $\left(\sigma_{y}(\boldsymbol{\sigma} \cdot \mathbf{p})\right)_{\alpha \beta}$ for $S=1$. For the broken 3D inversion near the surface, the Cooper pair wave function $\hat{F}$ becomes a mixture of singlet and triplet pairings. $\hat{F}_{\mathrm{s}}\left(\mathbf{p}, \omega_{n}\right)$ is the singlet part component, while $\hat{F}_{\text {as }}\left(\mathbf{p}, \omega_{n}\right)$ provides for the triplet admixture.

As an example of the phenomenon in which breaking the parity of the order parameter manifests itself, we calculate the spin susceptibility $\chi^{\mathrm{sp}}(T)$ of the surface SC state. It can be directly tested by Knight shift experiments. For systems with strong SO coupling, the total magnetic susceptibility $\chi$ cannot be split into the orbital and spin parts. However, when $\alpha \ll v_{F}$ such a division is justified [19], and we find $\chi^{\mathrm{sp}}$ neglecting the quantization of the orbital motion. Following Ref. [20], we calculate the tensor $\chi_{i j}^{\mathrm{sp}}$ $(i, j=x, y, z)$ as a linear spin response to the magnetic field $\mathbf{B}$

$$
\chi_{i j}^{\mathrm{sp}}=-\mu_{\mathrm{B}}^{2} T \nu\left(\varepsilon_{F}\right) \sum_{\omega_{n}} \int d \xi \operatorname{tr}\left\{\sigma_{i} \hat{G}\left(\mathbf{p}, \omega_{n}\right) \sigma_{j} \hat{G}\left(\mathbf{p}, \omega_{n}\right)-\sigma_{i} \hat{F}\left(\mathbf{p}, \omega_{n}\right) \sigma_{j} \hat{F}^{+}\left(\mathbf{p}, \omega_{n}\right)\right\} .
$$

Here the trace is taken over the spin indices, $\nu\left(\varepsilon_{F}\right)=$ $p_{F} / 2 \pi v_{F}$ is the density of states at the Fermi level (per one spin orientation), and $\mu_{\mathrm{B}}$ is the Bohr magneton. In the normal state the right hand side of Eq. (27) diverges because of Landau singularity [16], and to resolve it the summation over $\omega_{n}$ should be performed first. Finally

$$
\chi_{N}^{\mathrm{sp}}=2 \mu_{\mathrm{B}}^{2} \nu\left(\varepsilon_{F}\right) .
$$

Remarkably, $\chi_{N}^{\mathrm{sp}}$ found in the weak field limit, $\mu_{\mathrm{B}} B \ll$ $\alpha p_{F}$, when Zeeman splitting is small compared to the SO splitting, is isotropic, and coincides exactly with the Pauli susceptibility. Since the difference in $\chi^{\text {sp }}$ in the normal and SC states comes from the integration over the region of only about $\Delta(T)$ near $\varepsilon_{F}$, the appropriate integral converges. Therefore, in these terms the integration over $\xi$ 
can be performed first. Nondiagonal components of $\chi^{\mathrm{sp}}$ are equal to zero because of the $\mathrm{C}_{{ }_{\mathrm{V}}}$ symmetry, while for the diagonal components of $\chi^{\text {sp }}$ (in-plane component $\chi_{\|}^{\text {sp }}$ and perpendicular-to-the-plane component $\chi_{\perp}^{\mathrm{sp}}$ ) somewhat cumbersome calculations result in

$$
\begin{gathered}
\chi_{\perp}^{\mathrm{sp}}(T)=\chi_{N}^{\mathrm{sp}}\left\{1-\pi T \sum_{\omega_{n}} \frac{\Delta^{2}(T)}{\sqrt{\omega_{n}^{2}+\Delta^{2}(T)}}\right. \\
\left.\cdot \frac{1}{\omega_{n}^{2}+\Delta^{2}(T)+\left(\alpha p_{F}\right)^{2}}\right\}, \\
\chi_{\|}^{\mathrm{sp}}(T)=\frac{1}{2} \chi_{\perp}^{\mathrm{sp}}(T)+\frac{1}{2} \chi_{N}^{\mathrm{sp}}\left\{1-\sum_{\omega_{n}} \frac{\pi T \Delta^{2}(T)}{\left(\omega_{n}^{2}+\Delta^{2}\right)^{3 / 2}}\right\} .
\end{gathered}
$$

It follows from Eqs. (29) and (30) that $\chi_{\perp}^{\mathrm{sp}}(T) \neq \chi_{\|}^{\mathrm{sp}}(T)$ and $\chi_{\perp}^{\mathrm{sp}}(0), \quad \chi_{\|}^{\mathrm{sp}}(0) \neq 0$; i.e., spin susceptibility is anisotropic and does not turn into zero at $T=0$. We attribute this fact to the admixture of the triplet state to the $s$-type SC ground state because of the broken parity. For $\alpha p_{F} \ll \Delta(T)$, it follows from Eqs. (29) and (30) that $\chi_{\perp}^{\mathrm{sp}}(0) \approx \frac{2}{3}\left[\alpha p_{F} / \Delta(T)\right]^{2} \chi_{N}^{\mathrm{sp}}$ and $\chi_{\|}^{\mathrm{sp}}(0) \approx$ $\frac{1}{3}\left[\alpha p_{F} / \Delta(T)\right]^{2} \chi_{N}^{\mathrm{sp}}$. Hence, for $\alpha=0$ the BCS result $\chi_{\perp}^{\mathrm{sp}}(0)=\chi_{\|}^{\mathrm{sp}}(0)=0$ is recovered. In the opposite limit $\Delta(T) \ll \alpha p_{F}$, we get $\chi_{\perp}^{\mathrm{sp}}(0) \approx \chi_{N}^{\mathrm{sp}}$ and $\chi_{\|}^{\mathrm{sp}}(0) \approx \chi_{N}^{\mathrm{sp}} / 2$; hence, $\chi_{\perp}^{\mathrm{sp}}(T)$ is nearly $T$ independent while $\chi_{\|}^{\mathrm{sp}}(T)$ drops twice when $T$ changes from $T_{c}$ to $T=0$. Anisotropy of $\chi(T)$ in the SC state has already been inferred in Ref. [14]. Our expressions for both $\chi_{\perp}^{\mathrm{sp}}(T)$ and $\chi_{\|}^{\mathrm{sp}}(T)$ essentially differ from those of Ref. [14].

We expect mixed singlet-triplet states to arise in the electrical contact areas and influence proximity effects and spin injection phenomena basic for spintronics [21].

In conclusion, we have shown that spin-orbit interaction lifts the spin degeneracy for 2D (surface) superconductor resulting in the two gapped branches in the energy spectrum. Thermodynamics of such a state would be almost identical to a BCS-like superconductor. Because of the broken 3D inversion symmetry at the surface, the pair wave function is the mixture of singlet and triplet components. As a result, strong anisotropy appears in the spinsusceptibility tensor and the Knight shift. Splitting of two gapped branches in the momentum space depends on the strength of SO coupling. The latter can considerably exceed the SC scale $T_{c}$ to be observed by other means.

L.P.G. thanks Z. Fisk for discussions of a possibility to observe surface $\mathrm{SC}$ in tungsten bronzes in the field-effect-transistor geometry. His work was supported by NHMFL through the NSF Cooperative agreement No. DMR-9521035 and the state of Florida. E.I.R. acknowledges support from DARPA by the Office of Naval Research Grant No. N000140010819.

*Also at L. D. Landau Institute for Theoretical Physics, Russian Academy of Sciences, 117334 Moscow, Russia.

${ }^{\dagger}$ Also at Department of Physics, MIT, Cambridge, MA 02139.

Email address: erashba@mailaps.org.

[1] S. Reich and Y. Tsabba, Eur. Phys. J. B 9, 1 (1999); A. Shengelaya, S. Reich, Y. Tsabba, and A. Müller, Eur. Phys. J. B 12, 13 (1999); Y. Levi, O. Millo, A. Sharoni, Y. Tsabba, G. Leitus, and S. Reich, Europhys. Lett. 51, 564 (2000).

[2] J. H. Schön, Ch. Kloc, R. C. Haddon, and B. Battlog, Science 288, 656 (2000); J. H. Schön, Ch. Kloc, and B. Battlog, Nature (London) 406, 702 (2000).

[3] R. J. Elliott, Phys. Rev. 96, 280 (1954).

[4] G. E. Volovik and L. P. Gor'kov, JETP Lett. 39, 674 (1984); Sov. Phys. JETP 61, 843 (1985). For a review see L. P. Gor'kov, Sov. Sci. Rev. A Phys. 9, 1 (1987); M. Sigrist and K. Ueda, Rev. Mod. Phys. 63, 239 (1991).

[5] M. M. Salomaa and G. E. Volovik, Rev. Mod. Phys. 59, 533 (1987).

[6] H. Shimahara, Phys. Rev. B 62, 3524 (2000).

[7] M. Schultz, F. Heinrichs, U. Merkt, T. Colin, T. Scauli, and S. Løvold, Semicond. Sci. Technol. 11, 1168 (1996).

[8] J. Nitta, T. Akazaki, H. Takayanagi, and T. Enoki, Phys. Rev. Lett. 78, 1335 (1997).

[9] S. LaShell, B. A. McDougall, and E. Jensen, Phys. Rev. Lett. 77, 3419 (1996).

[10] E. Rotenberg, J. W. Chung, and S. D. Kevan, Phys. Rev. Lett. 82, 4066 (1999).

[11] E. I. Rashba, Sov. Phys. Solid State 2, 1109 (1960).

[12] Yu. A. Bychkov and E. I. Rashba, JETP Lett. 39, 78 (1984).

[13] L. Petersen and P. Hedegård, Surf. Sci. 459, 49 (2000).

[14] L. N. Bulaevskii, A. A. Guseinov, and A. I. Rusinov, Sov. Phys. JETP 44, 1243 (1976). However, we could not establish correspondence between their and our results.

[15] V. M. Edelstein, Phys. Rev. Lett. 75, 2004 (1995); JETP Lett. 69, 392 (1999), and references therein.

[16] A. A. Abrikosov, L. P. Gor'kov, and I. E. Dzyaloshnskii, Methods of Quantum Field Theory in Statistical Physics (Dover, New York, 1975).

[17] Green functions are diagonal in $\lambda$ for our model, and may stay diagonal even under more general conditions.

[18] L. P. Gor'kov, Sov. Phys. JETP 7, 505 (1958).

[19] I. I. Boiko and E. I. Rashba, Sov. Phys. Solid State 2, 1692 (1960).

[20] A. A. Abrikosov and L. P. Gor'kov, Sov. Phys. JETP 15, 752 (1962).

[21] R. J. Soulen, Jr., J. M. Byers, M. S. Osofsky, B. Nadgorny, T. Ambrose, S.F. Cheng, P. R. Broussard, C. T. Tanaka, J. Nowak, J. S. Moodera, A. Barry, and J. M. D. Coey, Science 282, 5386 (1998); S. A. Wolf, J. Supercond. 13, 195 (2000). 